\title{
ㄷำ1
}

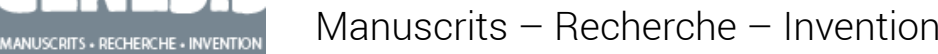

$37 \mid 2013$

Verbal - Non verbal

\section{Le laboratoire central de Robert Pinget}

Martin Mégevand

\section{OpenEdition}

\section{Journals}

Édition électronique

URL : http://journals.openedition.org/genesis/1246

DOI : 10.4000/genesis. 1246

ISSN : 2268-1590

\section{Éditeur :}

Presses universitaires de Paris Sorbonne (PUPS), Société internationale de génétique artistique littéraire et scientifique (SIGALES)

\section{Édition imprimée}

Date de publication : 15 décembre 2013

Pagination : 165-176

ISBN : 9782840509196

ISSN : 1167-5101

\section{Référence électronique}

Martin Mégevand, «Le laboratoire central de Robert Pinget », Genesis [En ligne], 37 | 2013, mis en ligne le 21 mars 2016, consulté le 21 janvier 2021. URL : http://journals.openedition.org/genesis/1246

DOI : https://doi.org/10.4000/genesis.1246 


\title{
Le laboratoire central de Robert Pinget
}

\author{
Martin Mégevand
}

\author{
Il juge indécente la curiosité qu'ont certains érudits \\ des retouches et ratures dans le manuscrit d' un auteur.
}

Robert PINGET, Le Harnais

$\mathrm{N}$ 'en déplaise à Monsieur Songe, à qui Pinget prête les mots cités ici en épigraphe, il peut valoir la peine de risquer l'indécence. De l'exploration des dossiers de genèse et des inédits de Robert Pinget mis désormais à la disposition des chercheurs à la Bibliothèque littéraire Jacques Doucet ${ }^{1}$, nous pouvons en effet espérer tirer au moins trois bénéfices évidents. Tout d'abord, on espère éclairer la partie éditée par l'étude des différents états des manuscrits mais aussi par celle des œuvres abandonnées afin d'établir une reconstitution des traits fondamentaux qui constituent le parcours esthétique de Robert Pinget. Il s'agirait moins de traquer certains aspects de la psychologie de la création, tels que les rôles joués par l'autocensure ou la sublimation, que de repérer les stratégies d'écrivain, notamment le rapport aux modèles et la défiance à l'égard des tournures empruntées. Le deuxième bénéfice attendu est de pouvoir composer un nouveau paysage de la production de Robert Pinget, où apparaissent de nouveaux liens logiques et formels entre les œuvres publiées : le nombre de textes de Pinget demeurés inédits est encore, à ce jour, considérable, puisqu'il se constitue au bas mot de sept dossiers de genèse d'épaisseur et de cohérence variables, pouvant aller jusqu'à plusieurs centaines de feuillets ${ }^{2}$. Le troisième bénéfice est lié aux deux premiers par un rapport de conséquence : il s'agit d'établir des hypothèses sur le « laboratoire central » de l'écrivain ${ }^{3}$. On se rappelle le vœu de Valéry rapporté par Madeleine Renouard et Jean-Claude Liéber à l'ouverture du Chantier Robert Pinget : «L'objet d'un vrai critique devrait être de découvrir quel problème l'auteur s'est posé (sans le savoir ou le sachant) et de chercher s'il l'a résolu ou non ${ }^{4}$.»

Cette proposition, mise au singulier par Valéry, est d'ores et déjà exploitée depuis que la psychanalyse prend la littérature pour objet. Elle se justifierait pour Pinget, du fait que l'écrivain l'induit lui-même dans sa manière de parler du travail d'écriture en termes de «drame » à élucider, à propos de sa collaboration avec Samuel Beckett :

[...] j'avais le sentiment d'être au cœur de son drame constitué de sensations et de sentiments d'une acuité morbide 5 .

Évoquer le travail d'un écrivain en termes de «drame» présente le risque d'une interprétation globalisante qui

1. Le fonds Robert Pinget est consultable à la Bibliothèque littéraire Jacques Doucet sous la cote intitulée PNG. Les manuscrits sont répertoriés sous les cotes PNG 1 à PNG 292.

2. Sept dossiers de genèse entièrement inédits à ce jour sont répertoriés dans Calames (<www.calames.abes.fr $>$ ), mais plusieurs textes inédits sont joints aux dossiers des textes publiés, et le répertoire est encore incomplet à ce jour.

3. L'expression est ici choisie pour rappeler l'importance de la référence à Max Jacob pour Pinget.

4. Paul Valéry, Tel Quel, Paris, Gallimard, coll. «Bibliothèque de la Pléiade », p. 539, cité par Liéber et Renouard, dans Le Chantier Robert Pinget, Paris, Jean-Michel Place, 2000, p. 33.

5. « Notre ami Samuel Beckett », p. 19, dactylogramme inédit déposé à la Bibliothèque littéraire Jacques Doucet, non coté à l'heure où nous écrivons cet article. Pinget a pu emprunter le terme à Sartre, qui l'emploie à propos de Baudelaire (Sartre, Baudelaire, Paris, NRF, coll. «Les essais », 1948, p. 28). Il avait acheté le Baudelaire de Sartre dans une édition de 1948, dont les thèses ont pu l'impressionner. 
reconduise aux apories du topos romantique du génie. Une approche qui prenne en compte faits esthétiques, données psychiques et sociologiques, rapport à l'idéologie et au savoir, peut s'appuyer sur des données génétiques sans recourir à la notion de drame intérieur, pour s'attacher à la question de la singularité d'écrire : ce sera celle proposée ici.

Saisir l'écriture de Pinget par le biais du singulier, c'est ouvrir sur d'autres problématiques que celle de la seule caractérisation d'un geste esthétique : c'est aussi demander avec quoi (ou éventuellement qui) l'œuvre de Pinget fait communauté, c'est-à-dire quel est son rapport au savoir, et de quelle manière elle s'inscrit dans la modernité.

Cette orientation peut à juste titre se situer dans l'horizon d'un travail génétique qui par définition se focalise sur le singulier, sans perdre de perspective la trajectoire d'ensemble de l'écrivain. La présente démarche pourrait se formuler de la façon suivante : quel sens les inédits et les états antérieurs des textes publiés peuvent-ils donner à la phrase «Robert devient écrivain »? Cette phrase s'entend selon trois sens, et recoupe trois ordres d'interrogations.

« Robert devient écrivain » peut se comprendre dans le sens d'une exigence toujours plus grande de perfectionnement : Robert devient un bon écrivain 6 . À titre d'illustration, on s'essaiera, par un prélèvement de type moléculaire, à observer les variations d'une phrase célèbre de Pinget présente dans le récit inédit «Fonfon » et modulée dans Passacaille, publié en 1969.

La phrase se saisit aussi dans le sens social d'une consécration, ou tout au moins d'une intégration dans la compagnie des écrivains d'un groupe bien repéré et délimité, et reconnu dans l'histoire littéraire sous l'appellation du « groupe de Minuit ». L'observation du travail d'écriture de Pinget permet-elle d'inférer des stratégies par lesquelles l'écrivain tenterait de se situer progressivement dans le champ de la création littéraire de son temps 7 ? À titre indicatif ici encore, on proposera des pistes de réponse à cette question en observant les chutes de Mahu ou le matériau, roman décisif pour l'intégration de Pinget dans le milieu littéraire des années cinquante et constituant à cet égard un document du plus grand intérêt.

Enfin, le troisième sens de «Robert devient écrivain 》 peut se gloser en « Robert devient toujours (ou perpétuelle- ment) écrivain » : il s'agit d'envisager l'œuvre dans son devenir, d'examiner à nouveau les traits de son évolution tout en tenant compte de l'exigence de renouvellement de l'écriture par Pinget qui, à chaque nouveau travail, tente de « tout reprendre à zéro 8 », ce qui le conduit justement à retracer des chemins déjà parcourus. Deux questions se posent ici : les œuvres inédites permettent-elles de faire apparaître des liens jusqu'ici inapparents entre les œuvres et les périodes, toujours contestables mais nécessaires ? Le texte inédit intitulé «Messire Jonas », par exemple, qui se situe à la croisée du théâtre, du récit épique, de la parodie du sermon et de l'invention, tératologique, fait signe vers bien des œuvres ultérieures dont Graal Flibuste, Baga, Cette voix. Ainsi, bien des parentés peuvent être décelées entre les textes non publiés et les œuvres bien connues de l'auteur, qui peuvent permettre de lever quelques obscurités propres à cette œuvre si multiforme sous une cohérence de surface. « Il a beaucoup écrit mais n'a soumis que certains textes à publication. Les raisons de ceci resteront obscures (luimême relisant certains fragments inédits en juin 1997 les trouvait aboutis) », témoignent Madeleine Renouard et JeanClaude Liéber ${ }^{9}$. Examinant « La Fissure ${ }^{10}$ », texte également non publié du vivant de Pinget, on posera des hypothèses sur cette mise à l'écart. La forme inachevée de la plupart de ces textes inédits les constitue moins en œuvres qu'en traces, ce qui permet de mettre en lumière des réflexes d'écriture relativement émancipés des opérations propres à la perspective de la publication, toujours source de tourments pour Pinget : «Mon Dieu, une publication! Devenir public, comme ça! », s'exclame le narrateur de Gibelotte 11 .

6. Une inquiétude qui ne le quittera pas, comme en témoigne le brouillon de l'avant-propos de « Psychophonie », roman abandonné datant de 1969 (PNG 150) : «J'aurai tout essayé. Mais peut-être après tant d'exercices, suis-je devenu un écrivain ? »

7. Sur ce sujet, voir le travail d'Aline Marchand, «Un nouveau poète à minuit. Ethos et horizons poétiques dans l'œuvre de Robert Pinget », thèse de doctorat sous la direction de Marc Dambre, Paris III, décembre 2012. 8. L'expression, de Michel Serre, est exploitée par Stephen Bann dans son article « L'Apocryphe ou la loi nouvelle », paru en traduction française dans la livraison de la Revue des Belles Lettres consacrée à Pinget, Genève, 1982-1. L'article est disponible sur le site Internet <www.robert-pinget. com>.

9. Dans Le Chantier Robert Pinget, op. cit., p. 21.

10. PNG 207.

11. Robert Pinget et Matias, Gibelotte, Paris, Jean-Michel Place, 1994. 
Le regard posé sur les textes inédits sera ici classé selon trois niveaux d'observation : celui, moléculaire, de la phrase, sera pris dans celui, élargi, de la séquence (récit, chapitre, séquence d'un roman) puis la perspective s'étendra au plan d'une œuvre envisagée dans son ensemble.

\section{Les manipulations moléculaires ${ }^{12}$}

Il a déjà été remarqué que la sélection des matériaux destinés à la publication est motivée par diverses procédures, parmi lesquelles l'autocensure et la sublimation ${ }^{13}$. L'opération de censure est également visible quand on compare les différentes versions de «Fonfon », récit inédit dont une version modifiée a été insérée par Pinget dans la dernière partie de Passacaille ${ }^{14}$. Fonfon est un jeune simple d'esprit qui apparaît dans Quelqu'un, où il est un hôte un peu à part de la pension que dirigent le narrateur et son compagnon. Une séquence importante du roman montrait déjà l'attention particulière portée par Pinget à ce personnage, par qui tout un rapport à l'imaginaire peut s'exprimer. La nouvelle inédite est centrée sur la relation ambiguë entre le narrateur et l'adolescent, occasion d'agacements multiples mais faite d'attentions, de prévenances et d'attachement sensuel. Ce que Pinget gomme, de «Fonfon » à Passacaille, ce sont les détails parfois très explicites portant sur la nature transgressive de la relation entre le maître et l'idiot, et sur les pratiques solitaires de ce dernier. Le texte de «Fonfon » est ainsi plus cru que celui publié dans Passacaille, comme en témoigne une allusion au sacrilège, soulignée par la cocasserie d'un détail sulpicien (le narrateur imagine Fonfon puis s'imagine lui-même se masturbant sous une image du Sacré-Cœur).

L'opération d'autocensure - qu'elle soit motivée par un contenu transgressif ou par la rupture de ton qu'induit l'insertion de la nouvelle dans le roman cible - constitue indéniablement un premier motif de correction de l'auteur : mais on remarque aussi diverses opérations d'ordre esthétique conduisant à une transformation parfois radicale du sens de la phrase initiale. À titre d'exemple, on propose de confronter deux versions d'une phrase célèbre écrite dans « Fonfon » puis publiée dans Passacaille. Trois opérations principales de transformation de cette phrase se repèrent : une intervention sur les dimensions du texte par réduction ou expansion; des procédures de reformulation et des opérations de déplacement.

«Fonfon », dactylogramme d'une trentaine de feuillets, raconte la relation entre le maître et l'idiot. Pinget y puise la matière d'un récit second de Passacaille, qu'il articule aux autres grands motifs du roman, notamment à l'intrigue policière et au travail d'écriture du maître. C'est dans ce récit second que se trouve la célèbre phrase :

Que nous n'ayons pas encore trouvé une phrase, depuis le temps, pour nous en passer de la nature, une phrase qui retienne tout ensemble, on la dirait le matin l'estomac plein jusqu'au soir où devant le coucher du soleil on la redirait la bouche pâteuse, plus besoin de sommeil ni de plaisir, phrase nourrissante, apaisante, la panacée, en désherbant le pré, en lavant le $\mathrm{Z}$ des autres, alimentaire, potable, éclairante, jusqu'au jour... (Passacaille, Minuit, 1969, p. 117).

En voici la version dans « Fonfon » (voir fig. 1, pour le contexte narratif de la phrase, qui commence au bas de la page) :

Que le malheur nous laisse toujours dépourvus, que nous n'ayons pas encore trouvé une phrase à effacer les larmes, depuis le temps. Une phrase qui veuille tout dire, qui retienne tout ensemble, plus besoin de se creuser la tête, plus besoin de comprendre ni d'aimer ni de consoler ni de rien faire, on la dirait le matin et nous voilà le cœur et l'estomac pleins jusqu'au soir où devant le coucher du soleil on la redirait la bouche pâteuse, plus besoin de plaisir, on se rendormirait jusqu'au lendemain, oui j'y pense, les chats pourraient bien crever, et les crétins jouir, et les confitures passer, notre sérénité serait la même, phrase nourrissante, apaisante, la panacée, en désherbant le pré, en guettant des visites, en lavant le $\mathrm{Z}$ des autres, alimentaire, potable, éclairante, jusqu'au jour où sur notre grabat nous la dirions une dernière fois pour voir s'ouvrir tout grand l'empyrée des Japonais...

Des trois opérations principales de transformation, la plus visible consiste dans la réduction du texte premier. Pinget gomme d'abord les allusions à des péripéties du récit

12. J'emprunte l'usage critique de cet adjectif à Michel Vinaver, dans Écritures dramatiques. Essais d' analyse de textes de théâtre, Arles, Actes Sud, 1993.

13. M. Renouard, J.-C. Liéber, op. cit., p. 19.

14. «Fonfon », PNG 127 
premier, en contextualisation immédiate - trouver une phrase à effacer les larmes, c'est consoler Fonfon qui pleure devant son chaton saigné par une fouine - ou plus éloignée : l'allusion aux confitures qui passent, à la jouissance du crétin et à l'empyrée des Japonais renvoient à des séquences antérieures du récit de «Fonfon ». Mais dans cette opération de réduction du texte source, c'est l'explicitation d'une axiologie qui disparait : la perspective de sérénité, l'image finale, ironique et grandiose, de l'espérance au cœur de l'agonie («pour voir s'ouvrir... ») qui rapprocherait peut-être trop visiblement le texte de la mort de Félicité dans Un coeur simple et du lexique beckettien par la présence du mot grabat. Enfin, plusieurs mots désignant des affects sont gommés (malheur, larmes, cœur). Par ces diverses suppressions, Pinget compose une épure qui neutralise le pathos, qui suggère au lieu d'expliciter, tout en conservant des références précises à la matérialité du corps (estomac, bouche, Z) et à la physiologie. Cette opération a donc pour résultat le maintien de la matérialité et l'ouverture du sens : les points de suspension laissent la période ouverte, inachevée : "jusqu'au jour... ». Elle conduit aussi à effacer la dimension narrative pour orienter de manière décidée le texte vers le poème en prose. De la sorte, par cette seule opération de suppression, apparaît un aspect fondamental du travail d'autoréécriture de Pinget : abandonnant la narrativité et l'explicitation, il ouvre la phrase vers une indécidabilité du sens.

Mais il y a plus. Le second texte n'est pas seulement une réorientation formelle et poétique du texte premier : il en est aussi la contradiction. S'ajoutant aux opérations visant à réduire ou étendre le texte premier, une troisième opération consiste à reformuler de façon contradictoire la première version. Où le sommeil est espéré dans le texte premier (« on se rendormirait jusqu'au lendemain »), la deuxième version reformule : «plus besoin de sommeil ». Le désir d'abrutissement dans «Fonfon » s'inverse dans Passacaille en désir de demeurer en éveil - et c'est alors tout le sens de la phrase qui s'inverse. Raccourcie, la phrase rapproche des signifiants : sommeil - soleil - plaisir - apaisante. Le mot sérénité a beau avoir été gommé du second texte, il continue de se présenter à l'esprit du lecteur, par les seuls effets du rythme et des paronomases. C'est que Pinget, dans une quatrième opération, a déplacé : il s'est laissé guider par les sonorités et a ressaisi rythmiquement la période initiale, laissant en conséquence le sens dans l'approximation et l'ouverture. Les déclarations de Pinget sur l'accueil des contradictions ne se comprennent donc pas seulement sur le plan thématique; elles touchent l'acte même, minimaliste, d'assembler les mots, de corriger, de reprendre, de redire.

En somme, le texte publié est plus inachevé que le texte premier ; le geste esthétique vise à soustraire, retrancher : l'inachèvement devient un objectif, le signe d'aboutissement de l'œuvre. C'est pourquoi la phrase de «Fonfon » ne saurait aider à éclairer le sens de celle de Passacaille, puisque Pinget se saisit de la première version comme d'un matériau à transformer.

Par la comparaison des deux versions d'une même phrase apparaissent ainsi des gestes esthétiques fondamentaux : l'intervention de Pinget sur son texte peut être qualifiée à la fois comme le geste d'un poète qui infléchit un texte romanesque vers sa «transformation élocutoire », comme celui d'un peintre qui travaille son esquisse en gommant ses contours, et comme celui d'un moraliste qui transforme un texte en maxime. On aura reconnu trois dimensions principales de l'œuvre de Robert Pinget, qui apparaissent comme des constantes de son écriture.

Il serait cependant insuffisant de limiter le passage à la publication à cet ensemble de procédures d'ordre psychique ou esthétique ${ }^{15}$. Une stratégie de négociation avec des impératifs extérieurs, notamment éditoriaux, est aussi à l'œuvre, dont les chutes de Mahu rassemblées dans le dossier de genèse intitulé «L'Anti Mahu » permettent de rendre compte, au moins pour partie.

\section{Les interventions sur les séquences ${ }^{16}$}

Onze nouvelles (ou poèmes en prose inédits), présentant une cohérence de projet et de style, composent les

15. Ceci peut expliquer par exemple qu'un texte travaillé mais très fantasmatique comme «Psychophonie » (PNG 141 à 150) ait été finalement abandonné par Pinget ; voir Robert Pinget : matériau, marges, écritures, dir. Martin Mégevand et Nathalie Piégay-Gros, Saint-Denis, Presses universitaires de Vincennes, 2011.

16. On propose de nommer «niveau séquentiel » un niveau intermédiaire entre la phrase et l'œuvre : chapitres, nouvelles, récits secondaires. 


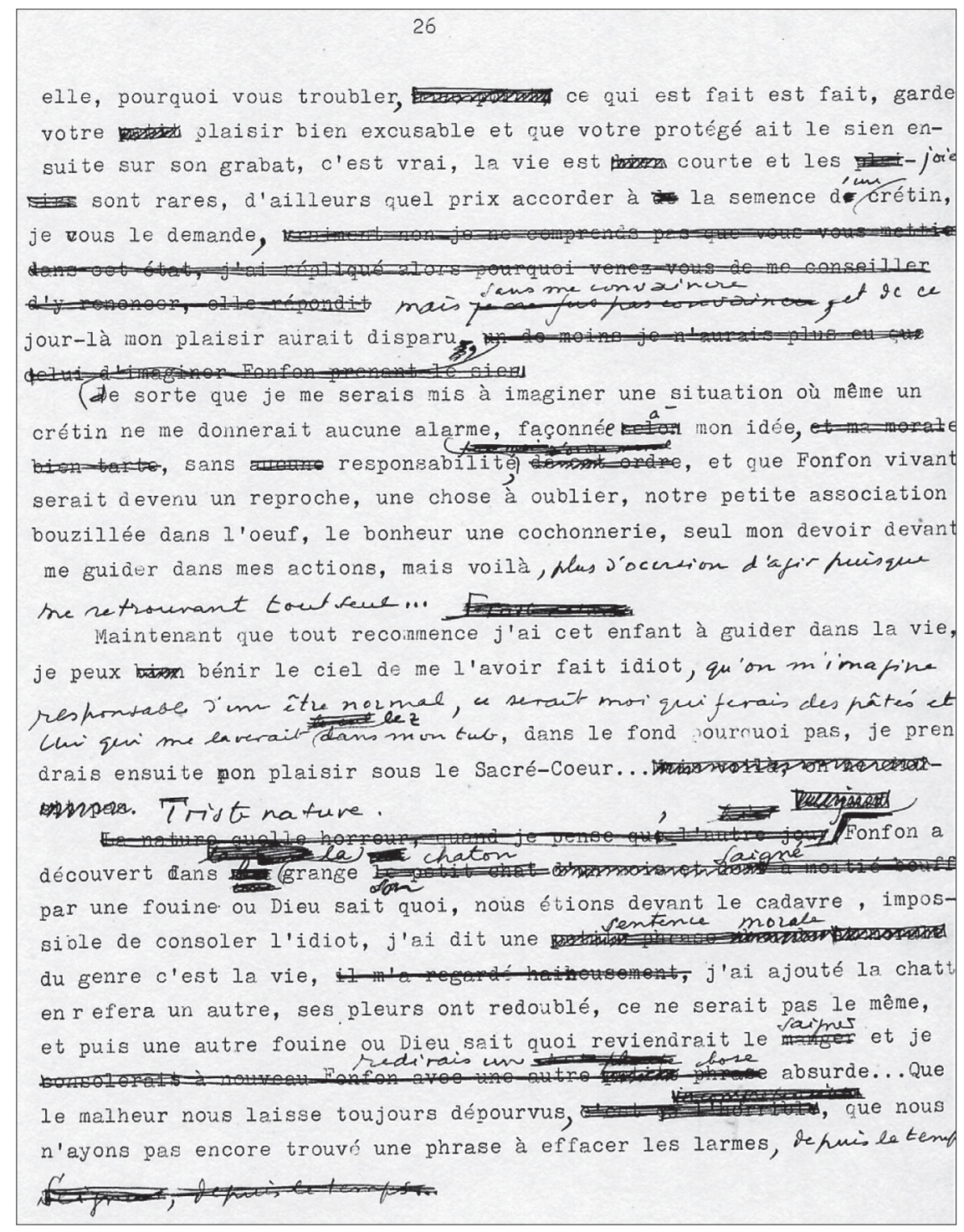

Fig. $1:$ : Fonfon », tapuscrit corrigé

(Chancellerie des Universités de Paris, Bibliothèque littéraire Jacques Doucet, PNG 127, f. 26)

chutes de Mahu ou le matériau. À première vue, deux caractéristiques les distinguent des autres nouvelles retenues dans le recueil publié Mahu ou le matériau: la présence marquée de la référence, et la dimension de satire sociale.

À la première lecture, la présence d'un référentiel anecdotique paraît beaucoup plus massive que dans les nouvelles publiées. Ainsi, un fait divers historique, celui de la mort du roi d'Angleterre Georges VI survenue alors que sa fille Elizabeth accomplissait un safari au Kenya, est évoqué dans deux des nouvelles mises de côté par Pinget. Cette anecdote que Pinget retrace avec cocasserie eut un certain retentissement dans les journaux de l'époque. Si elle permet une datation précise de l'écriture de ces nouvelles (après le 5 février 1952, et avant la publication de Mahu ou le matériau, à l'automne de la même année), c'est sa suppression qui est signifiante : dès l'aube de l'œuvre, les qualités d'observation, d'ironie et d'humour de Pinget ne seront pas placées au service d'une description de la comédie humaine. Pinget se dégage de la référence contemporaine dès lors qu'elle est trop précise. Cet effacement de l'actualité s'accompagne du gommage des références topographiques et toponymiques qui localisent précisément les nouvelles dans Paris. Si Paris est localisé dans Mahu, c'est par des allusions vagues au métro, par exemple, alors que dans les nouvelles non publiées apparaissent le faubourg Saint-Denis, le passage Brady, le quai des Orfèvres. 
Ce ne sont pas seulement les coordonnées trop précises de l'espace et du temps qui se trouvent écartées du livre publié : le trait différentiel principal des nouvelles écartées est leur tonalité de satire sociale. Ces onze nouvelles mettent en scène des petites anecdotes constituées de trois à six personnages, Mahu étant accompagné de trois couples, Latirail et Ninette, Milane et Sofia, Louis et sa "bonne amie infirmière aux dents blanches », Mahu formant lui-même couple avec la concierge. L'orientation sociale de ces nouvelles est plus nettement puisée dans l'événementiel autobiographique que dans les nouvelles publiées : on y repère le passage de Pinget par l'école des beaux-arts, les soirées arrosées, les relations amicales estudiantines qui composent le cliché d'un environnement " bohème ». Les supprimer de Mahu ou le matériau, c'est procéder à une forme d'effacement et choisir la discrétion. De même que Pinget a renoncé à publier Jean Loiseau, roman qu'il qualifiait d'inévitable essai autobiographique, il s'abstient de rendre publiques ces nouvelles, prévenant ainsi tout risque d'étiquetage du satiriste; gommant la référence trop directe, qu'elle soit autobiographique ou topographique, il recentre son écriture sur la question du langage.

L'effacement de la référentialité parisienne peut se comprendre d'au moins trois manières, selon qu'on privilégie l'histoire littéraire, une stratégie de positionnement social ou une préoccupation esthétique.

Il peut s'agir d'une façon de se distinguer : ne pas écrire dans une manière déjà explorée par ces arpenteurs célèbres de Paris qu'étaient les surréalistes, dont Pinget récusera toujours l'influence sur son travail. Mais aussi, cette opération de gommage de références n'est peut-être pas sans rapport avec une position de marginalité dans le monde artistique parisien : Pinget est poète chez les peintres, peintre chez les poètes, avocat chez les artistes ; son intégration dans la vie parisienne n'est pas si solide qu'il puisse ainsi la manifester dans son œuvre, contrairement à un Max Jacob qui, lui, vivait au cœur de l'art moderne et ne se faisait pas faute de le signaler dans ses textes. Pinget est encore un «provincial » récemment arrivé à Paris à l'époque de $M a h u$, et ne revendiquera publiquement la paternité de Max Jacob qu'à partir des années soixante, lorsqu'il sera devenu un écrivain reconnu par la consécration du prix des Critiques en 1962. Or il est clair que l'au- teur du Cabinet noir apparaît à Pinget comme un modèle d'intégration réussie dans le monde parisien des arts ${ }^{17}$.

L'examen des chutes de Mahu permet également de penser la fameuse « unité de surface » que Pinget déclare tenter d'établir dans ses textes depuis Entre Fantoine et Agapa. L'expression, qui renvoie à l'univers des arts plastiques, pourrait faire croire à une unité constituée visuellement, sur les bases par exemple de cette topographie campagnarde labyrinthique qui prévaut dans L'Inquisitoire et dans tant d'autres romans. Or les chutes de Mahu mettent le lecteur sur une autre piste : s'il y a recherche d'une unité de surface dans $M a h u$, force est de constater qu'elle se fait au prix d'un gommage des repérages visuels, comme pour laisser l'oreille orienter la lecture. Surtout, cette unité de surface est opérée à la faveur d'un recentrement sur la figure centrale et rayonnante qu'est Mahu. En effet, ne pas retenir les nouvelles qui se singularisent par leur cohérence thématique, décrivant un Mahu socialisé, c'est renoncer à la cohérence narrative assurée par la description d'un milieu, au profit du coq à l'âne, de stratégies de ruptures et de liaisons obliques entre des histoires thématiquement hétérogènes. C'est se permettre cette « extraordinaire merveille qu'est un roman raté dans les grandes largeurs », selon la formule célèbre du Renard et la boussole au sens où s'il y a volonté délibérée de rupture avec les codes romanesques, c'est précisément pour bien raconter, c'està-dire en privilégiant la varietas sur la continuité, les effets de surprise et de vitesse sur la lenteur des développements suivis.

De fait, donner l'effet de la rapidité à la lecture est une préoccupation constante de Pinget (voir l'incipit de Théo ou le temps neuf: " vite l'écrire, vite l'écrire, sa petite musique suivra le trajet de la plume»). Dans la nouvelle «Les carcasses », que Pinget renonce à joindre au recueil, le narrateur déplore, alors que les personnages chantent des chansons démodées : "Latirail allait trop lentement il traîne toujours sur ce qu'il aime moi c'est le contraire plus j'aime plus j'écourte je n'approfondis rien ma profondeur elle est dans le choc que je ressens il me creuse ${ }^{18}$. » Il y a

17. Je renvoie à mon article « Robert Pinget, Max Jacob, les inclassables de la modernité », Les Cahiers de l'association Max Jacob, $\mathrm{n}^{\circ}$ 11-12, oct. 2012, p. 107-117.

18. PNG 19 , f. 14 
là un trait constant de l'écriture de Pinget, par-delà les différents styles et genres auxquels il s'essaiera.

Première conclusion : aux opérations qui ont pour objet d' « inachever » l'œuvre s'ajoute un gommage des références et un démarquage à l'égard des présupposés propres aux genres, comme pour décourager à l'avance toute tentative de rattachement des textes à un canon littéraire préétabli. Pinget démontre qu'écrire, c'est s'affranchir des attendus du genre auquel se rattache le texte qu'il écrit et choisir ses propres contraintes. C'est pourquoi il surprend toujours ses lecteurs, d'une œuvre à l'autre. Parmi les contraintes qu'il s'impose figure en bonne place le choix d'un rythme.

\section{Élargissement de la focale}

\section{La binarité de Mahu ou le matériau}

Comparer, en élargissant la focale, l'ensemble des différents états de Mahu ou le matériau permet de poser des hypothèses sur le rapport de Pinget au rythme. La structuration de Mahu ou le matériau est en deux parties, la première étant titrée « le romancier » et la seconde «Mahu bafouille », et inaugure les compositions binaires qui rythment la production de Pinget, au théâtre, de Lettre morte à Paralchimie, et dans ses textes romanesques comme Le Fiston, puis durant la décade des années soixante-dix, entre les colloques de Cerisy et de New York ${ }^{19}$.

Le narrateur de Mahu, à l'explicit du livre, qualifie la première partie de « roman raté », ce qui ne veut pas dire, pour autant, que la seconde partie serait un « roman réussi ». Un regard sur le dossier de genèse de Mahu renseigne sur le dispositif binaire qui régit le roman depuis son origine. On apprend que la première partie du roman était initialement titrée «l'engrenage ». Refuser ce terme, c'est récuser la métaphore mécanique au profit d'un paradigme beaucoup plus général, «le romancier », qui signale un projet et une volonté de contrôle de la narration, et annonce dès lors le ratage. Mais l'on constate que, dans la perspective de la publication, plusieurs nouvelles ont été déplacées de la première vers la seconde partie et inversement. Si une mise en ordre thématique apparaît, elle reste ébauchée : trois nouvelles concernant Latirail occupent, juste après la moitié de la première partie, les positions 14 à 16 sur les vingt-six nouvelles, mais elles forment comme un hapax dans l'œuvre. S'il y a un vague effet de mise en ordre, ce dernier est si peu contraignant qu'on se trouve conduit à conclure que la séparation entre les deux parties n'est pas d'ordre thématique mais d'ordre rythmique.

La division binaire est en effet d'abord une grande césure, et en ce sens peut se comprendre comme une variante de la ponctuation, au sens large que Véronique Dahlet donne à ce terme, d'une sorte de grande respiration ménagée au cœur du livre20. Les chutes de Mahu, qui forment une unité thématique et risquent de constituer un bloc hétérogène dans l'ensemble du recueil, paraissent confirmer cette hypothèse. En tout état de cause, cette césure respiratoire introduit une faille au cœur de la composition, qui partage le livre en deux ensembles souplement reliés par le style et par la focalisation sur le personnage de Mahu. De la sorte, le partage n'exclut pas toutes sortes de rapports entre les deux parties, au contraire, il favorise les rapprochements, mais peut se comprendre aussi comme la manifestation d'un refus d'une composition de type dialectique. La logique structurale de Mahu n'est pas celle, narrative, d'une évolution vers la résolution d'une crise. Elle obéit à un impératif pneumonique : ce que Pinget cherche avant tout à éviter, c'est l'asphyxie, la «bronchite »- ainsi qu'il le formule à l'explicit du recueil :

La première partie, entre nous, c'est un roman raté mais aucune importance, elle m'a servi de gammes préliminaires, ce qui m'importe ce n'est pas de bien chanter, c'est d'entendre une voix sans la bronchite, tu sais la bronchite il y a plein de petits sifflets.

On comprend que Beckett, pour qui écrire c'est essayer de respirer mieux ${ }^{21}$, ait volontiers écrit dans sa première lettre à Pinget qu'il avait « beaucoup aimé $M a h u$ ». S'il y a une contrainte liée au rythme, c'est celle que lui dicte son besoin de respirer.

19. Une dimension bien analysée par Aline Marchand dans sa thèse déjà citée. Voir le chapitre IV, p. 300 à 382.

20. Véronique Dahlet, De la ponctuation au ton : les romans de Pinget, thèse de doctorat, sciences des textes et documents, sous la direction de Julia Kristeva, Paris VII, 1990.

21. C'est l'hypothèse forte de Ludovic Janvier sur la motivation fondamentale de 1'écriture chez Beckett, énoncée notamment dans la revue Littérature, $\mathrm{n}^{\circ}$ 167, « Samuel Beckett », sept. 2012, p. 21 et 22. 


\section{Sur une æuvre écartée : La Fissure}

Mais comment comprendre au juste cette question de la respiration ? De quelle métaphore s'agit-il ici-s'il s'agit bien d'une métaphore, tant il est avéré que Pinget ne pouvait pas ne pas sinon écrire en tout cas inscrire, comme en témoigne l'abondance de ses textes abandonnés, des fragments griffonnés, des collages assemblés l'année de sa mort, et des formes courtes que les archivistes ont peine aujourd'hui à rassembler et à classer. On s'intéressera, pour tenter d'aborder ce point, à un texte abandonné intitulé $L a$ Fissure (1973-1975).

La Fissure est un texte limite, que Clothilde Roullier a édité chez MétisPresses en 200922. Dans sa préface très précise, elle qualifie le projet de tentative de «stéréophonie quadrifide ». Ce texte de quatre-vingt-un feuillets, dont la composition remonte selon Clothilde Roullier aux années 1973-1975, figure la recherche d'un équilibre géométrique 23 . Pinget, qui en abandonne la publication, en recycle les matériaux textuels dans d'autres projets : pièces radiophoniques et composition du roman Cette voix, publié en 1975. Par différence, le projet peut s'éclairer s'il est rapproché d'une œuvre singulière de Pinget, qui fut menée jusqu'à la publication : le petit livre d'art intitulé Cette chose.

Cette chose, publié en 1967, est constitué d'un dialogue entre le plasticien Jean Deyrolle et le poète Robert Pinget : à chaque dessin du peintre correspond un poème qui le glose, y soulignant les traces d'une lutte entre deux forces antagonistes, d'un agôn qui aboutira à l'échec de la tentative d'équilibre entre ces deux forces. La Fissure n'obéit pas à cette même logique dynamique. Quatre blocs textuels disposés en vis-à-vis sur les pages laissent apparaître la forme d'une croix, qui disparaîtra dans les derniers feuillets au profit d'un partage en deux colonnes.

Le dispositif binaire de La Fissure signale ainsi de manière singulièrement nette que le texte renvoie à une symbolique; c'est peut-être la grande visibilité symbolique du dispositif qui explique que Pinget ait renoncé à publier ce texte. Mais surtout, l'idée formelle, pour ingénieuse qu'elle soit, semble bloquer le développement narratif. Cherchant à aérer son texte en pratiquant des blancs, Pinget paraît s'asphyxier, et abandonne. La recherche d'une respiration, qui rapproche fondamentalement les entreprises de Pinget et de Beckett et qui les distingue - Beckett suppor- tant nettement plus longtemps l'état anaérobie que Pinget -, ne fonctionne pas pour ce texte. L'idée formelle se fige en procédé et Pinget, conscient du danger, abandonne son projet et en recycle les matériaux. Telle serait en tout cas la fiction théorique qui permettrait de rendre compte de ces abandons de Pinget, mais aussi de la proximité entre lui et Beckett : une même conception de l'écriture qui prend en compte leurs effets directs sur le corps de celui qui écrit et de celui qui lit, comme le confirme ce passage du dactylogramme inédit « Notre ami Samuel Beckett », où Pinget rend compte à son ami de sa lecture en tapuscrit de Comment c' est :

[...] il m'offre un whisky, puis un second. Je lui dis qu'il me doit bien ça après m'avoir mis à une telle épreuve avec son texte. J'avais chialé en le lisant, de rage et de dépit. Dans la troisième partie un certain lyrisme se dégage de ces nombres. Me dit que selon lui c'est la partie la plus faible. Je suis d'accord, mais on y respire mieux. Le halètement des deux premières est insupportable.

Qu'est-ce donc au juste que cet usage métaphorique de « respirer»? Entendons, avec Henri Meschonnic, que cela revient à rapprocher les mouvements du langage de ceux du corps. Ainsi de l'oralité :

\section{L'oralité ce n'est pas du son qu'on entend, comme dans le dualisme du signe (du son et du sens, de la forme et du contenu), c'est du sujet qu'on entend. C'est du sujet qu'elle vous fait. \\ Ainsi, l'oralité, c'est l'écriture, au sens poétique du mot, et le paradoxe de l'écriture, c'est qu'elle est la matière même de l'oralité. Sa réalisation maximale. À ne plus confondre avec le parlé. À ne plus réduire à la seule ponctuation 24 .}

S'intéresser aux tentatives abandonnées, c'est se donner la possibilité de comprendre les impossibilités d'écrire : Pinget expérimente sans cesse de nouvelles formes, et s'il se trouve parfois « coincé », c'est parce que le type de

22. Robert Pinget, La Fissure, précédé de Malicotte la Frontière, Genève, MétisPresses, 2009.

23. Je renvoie à la présentation de ce texte par Clothilde Roullier, op . cit., p. 11-16.

24. Henri Meschonnic, « Traduire le théâtre c'est traduire l'oralité », dans Bruno Curatolo (dir.), Traduire Lagarce, langue, culture, imaginaire, Besançon, Les Solitaires intempestifs, 2008. 
contrainte choisi éloigne trop le langage des contraintes dictées par le corps. Pinget dit écrire avec l'oreille : la formule, si connue, peut ainsi s'éclairer. Il s'agirait de la nécessité proclamée d'inscrire le sujet dans l'écriture, les mouvements du corps dans le langage, mais aussi ce que le savoir a imprimé dans sa mémoire.

\section{Savoir et mémoire : "Messire Jonas »}

Dans Gibelotte, qui est à la fois un art poétique et un court récit de vie, l'épistolier éponyme informe sa destinataire sur sa formation :

J'ai grandi dans cette atmosphère bêbête, mais que je regrette secrètement, jusqu' au jour où j'ai pris conscience de moi. Eh oui. Je me suis senti attiré par le savoir. [...] J'ai découvert que j'aimais beaucoup les musées et j'y ai passé les meilleures années de ma jeunesse. J'en connaissais plusieurs. Et surtout les jardins. J'adore les jardins, c'est plein de coins poétiques. On peut y réfléchir en paix à l'histoire, aux événements, à tout. J'y ai même tellement réfléchi, un temps, que les grands hommes du passé me visitaient la nuit.

L'acquisition du savoir est l'aliment d'une méditation, et celle-ci conduit à son tour à la vision et à l'hallucination. La lettre de Gibelotte à sa cousine détaille ensuite des phases hallucinatoires et métamorphiques du sujet. Ainsi, dans ce texte fondamental, c'est la libido sciendi qui, peuplant l'imaginaire du poète, conduit celui-ci à des expériences de désubjectivation, de métamorphose et de délire.

Ce que Gibelotte ne précise pas, cependant, c'est le contenu de ce savoir : qui sont ces grands hommes qui le visitent la nuit? On songe aux grands artistes, sans doute, mais aussi à ces figures de maîtres de la parole que sont les prophètes dont les paroles sont gravées dans sa mémoire depuis l'enfance.

C'est un inédit singulier, daté selon le classement de la Bibliothèque Jacques Doucet du début des années cinquante, qui articule le plus clairement un projet de fiction au substrat religieux de l'œuvre de Pinget. « Messire Jonas » est un dactylogramme de cent trente et un feuillets qui, constituant un avant-texte de Graal Flibuste (publié en 1956), pourrait avoir été écrit après Mahu ou le matériau (1952) et porte à la fois les traces de ses connaissances culturelles latines, acquises au Collège Calvin de Genève dans l'adolescence, de ses connaissances en histoire de l'art, et de sa formation religieuse (fig. 2). Ce texte est un hommage, unique dans l'œuvre de Pinget, à un prophète biblique. Il est dépourvu d'assignation générique précise : il s'approche du genre dramatique, mais la qualification de « moralité », bien présente dans le manuscrit, est effacée du dactylogramme. Sa forme relève davantage de la glose biblique, du sermon, du catalogue imaginaire et du journal intime que du théâtre. Dans ce texte foisonnant mais rythmé par des blancs qui aèrent presque toutes les pages, le travail d'écriture s'appuie sur un savoir hérité des années de formation : la littérature classique romaine et la Bible. Il est organisé en trois parties.

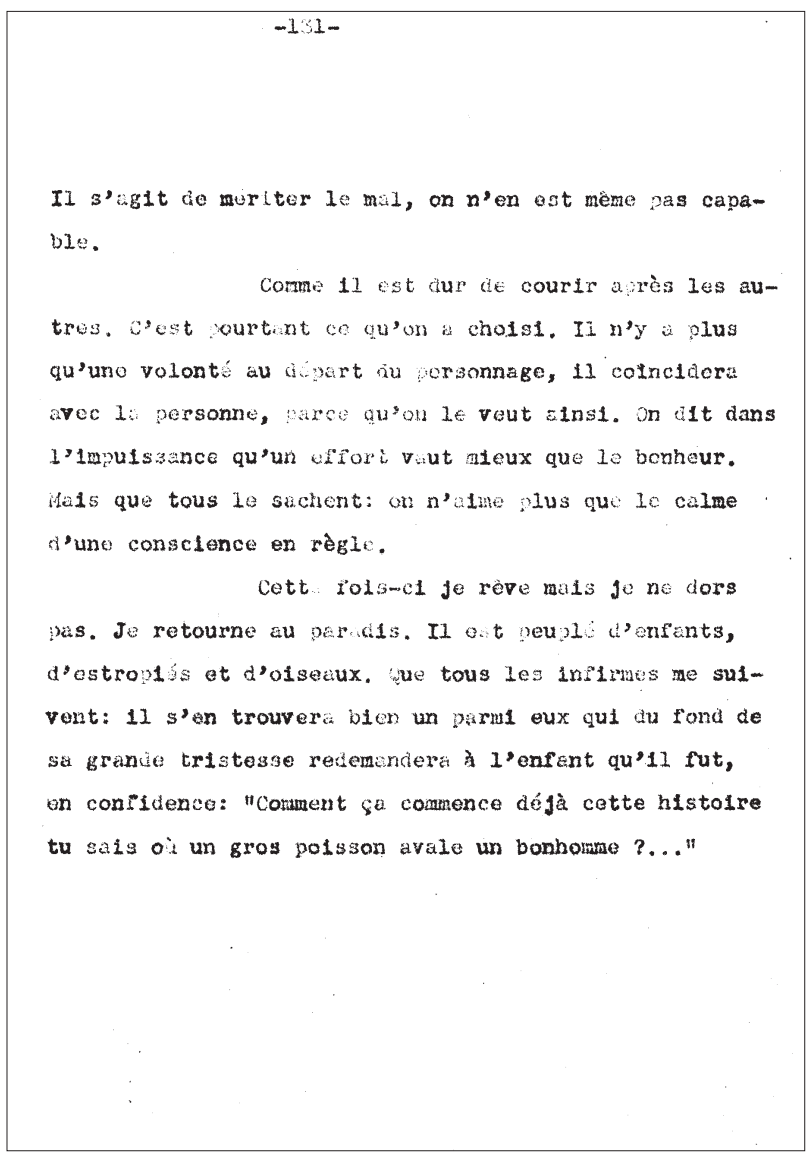

Fig. 2 : «Messire Jonas », dernière page du tapuscrit (Chancellerie des Universités de Paris,

Bibliothèque littéraire Jacques Doucet, PNG 40, f. 131) 
Un prêcheur anonyme commence par lire l'épisode biblique de Jonas, puis en effectue un commentaire segmenté, isolant dans le texte une proposition et en développant le commentaire («va à Ninive », ou « il se fit un grand vent », par exemple). Ce prêcheur y apparaît « en robe de bure et en chapeau de fou plein de grelots. Il a des lunettes de carton sur le ventre ». Le texte, presque dépourvu de didascalies après cette brève indication initiale, est pour sa première partie constitué d'un commentaire du texte biblique émaillé de références érudites mais aussi de notations d'humeur déployant un topos sur le désenchantement du monde d'aujourd'hui. Mais ce topos se trouve revitalisé au cours du texte. Le prêcheur ne se contente pas, en effet, de vitupérer l'époque. Ce qu'il déplore, c'est qu'on ne croie plus aux monstres. Dans une deuxième partie, intitulée "Les acteurs », ces monstres regrettés reçoivent la parole. Le chat-tigre, le bateau, Ninive, les animaux morts, le faux prophète, la muse : chacun s'exprime, se présente. Une courte troisième partie prend la forme d'une réflexion critique qui récuse la dimension morale de la légende de Jonas pour insister sur le vacillement identitaire, la fable étant comprise comme une allégorie non de la condition humaine mais de la condition d'artiste, qui postule l'accueil des contradictions morales (faire l'ange et la bête), résumé par la formule : «l'accomplissement de soi comme une bouteille glou-glou $25 »$.

Sur cette trame, l'orateur développe une méditation à la fois métaphysique et concrète sur le rapport à deux types de manifestations de l'altérité - le petit autre et le grand autre : la figure de l'homme, sous divers avatars dégradés (les mariniers et la figure de Levent, l'importun ${ }^{26}$ ), et Dieu, que Pinget nomme « Machin », les deux figures se confondant.

Cette méditation prend appui sur le texte biblique pour proposer d'abord une conception très personnelle de la résurrection chrétienne, venant ensuite à la déploration du désenchantement du monde auquel l'orateur oppose une série de références classiques et néoclassiques, citant Pline, Méla, Solinus, Transylvanus. La première partie de « Messire Jonas » passe par une méditation sur les représentations mythologiques de la lutte morale :

Il n'y a pas de pureté sans les hiboux mammelus, les singes à trois sexes, les papillons phalliques, Saint Antoine était houspillé par des sorcières à poil et des garçons équivoques et des vers énormes, pleins d'oreilles, que Breughel et Jérôme Bosch ont magnifiquement représentés et qui symbolisent la lutte, ils ne symbolisent pas ils sont la tentation visible en pleine nuit, les animaux horribles et familiers du Bien et du Mal. Tant qu'ils existent il y a combat.

La méditation ménage un accueil généreux à la littérature classique profane et (déjà) au savoir alchimique, au service d'un développement qui devient un plaidoyer en faveur de la fantasmagorie et de son efficacité pratique :

[...] autant d'images, autant de prières et de frayeurs, voilà leur fin du monde à ces pauvres mariniers, voilà leurs dieux. Ils appellent à la rescousse des visions d'enfer parce qu'ils n'ont pas appris à secouer leur paillasse le matin, ils retrouvent le soir un dortoir puant où ils s'endorment comme des brutes.

Dans sa désinvolture même, ce texte constitue une catharsis des convictions antérieures de Pinget, telles qu'elles se donnent à lire dans le journal intime de 1944, qui laisse apparaître un «fou de Dieu » extasié par la Pentecôte, égaré dans des spéculations abstraites. L'orientation spirituelle s'ancre ici dans le concret d'un jeu avec l'interlocuteur anonyme et avec ce grand Autre désacralisé par le détournement du dogme religieux.

Le projet de « Messire Jonas », qui est aux antipodes de l'apologétique, contient ainsi une réappropriation singulière de la lecture chrétienne du livre de Jonas, qui y voit une préfiguration de la Résurrection du Christ : la méditation du narrateur transforme cette interprétation dans le sens d'une conception de l'activité de l'esprit :

[...] on n'arrête pas de repêcher quelque chose qui allait se noyer, ou qui était noyé depuis longtemps. Nos pensées, comme tous les gens et les objets qui nous entourent, ne sont vivantes qu'une fois tuées, mortes d'oubli ou de malheur, elles renaissent par inadvertance dans l'esprit transitoire qui finit par vivre de leur éternité ratée27.

25. Ce motif sera repris dans l'avant-propos de Graal Flibuste, dont « Messire Jonas » est à bien des égards un avant-texte.

26. Un extrait de ce texte, centré sur la figure de l'importun, a été publié dans Europe, $\mathrm{n}^{\circ}$ 897-898, janvier-février 2004, sous le titre Levent (raisonneur omniprésent), p. 26-29.

27. « Messire Jonas », PNG 40, p. 11. 
La forme d'affranchissement de Pinget à l'égard du dogme religieux est ici intégrale. S'affrontant librement au texte biblique, il en réaménage la leçon morale à son usage. $\mathrm{Ce}$ que montre ce texte où les notations triviales se mêlent aux citations savantes, c'est que Pinget éprouve le besoin de faire tenir ensemble des données, radicalement hétérogènes, de l'expérience et du désir, telles que recentrement sur soi et ouverture à l'altérité, désir de peindre et goût des mots, littérature et art, soumission aux maîtres anciens et émancipation des modèles.

Enfin, « Messire Jonas » montre de manière très claire une mise en tension de l'écriture entre deux pôles : celui, aristocratique et solitaire, de l'attachement aux formes les plus élaborées du savoir et celui, démocratique et familier, du goût pour l'appel à la confidence et à la complicité du lecteur et pour les formes les plus archaïques de figuration de la relation à l'autre que garantit, dans ses fictions les plus célèbres, la figure de l'idiot.

La lecture de « Messire Jonas » permet de comprendre certains échecs et ratages de l'œuvre. Le dactylogramme montre que l'écriture est chez Pinget affaire de composition spatiale, où ces espaces de respiration que sont les blancs renvoient à l'expérience du sujet écrivant, comme l'étude des structurations de Mahu ou le matériau l'a confirmé. L'examen de « Messire Jonas » permet en outre de conforter des hypothèses sur le rapport au savoir qu'entretenait Robert Pinget, et qu'il confiera obliquement à Gibelotte, l'épistolier, plusieurs années plus tard. Ce savoir est ancré dans les textes sacrés et profanes de la tradition occidentale la plus ancienne ; il s'agit de les revisiter pour solliciter l'imaginaire dans une lutte contre le désenchantement qui constitue sans doute le cœur de sa singularité d'écrivain. Mais Pinget déposera légèrement ce savoir dans son œuvre publiée, manifestant une constante défiance à l'égard de l'étalage d'érudition, travers auquel il cède dans « Messire Jonas » et qui peut suffire à expliquer qu'il ait fini par l'abandonner.

Incontestablement, une lecture critique des documents disponibles à Doucet peut aider à mieux saisir les paradoxes de ce parcours d'écriture qui essaie constamment de nouer des inconciliables. Jean-Claude Liéber et Madeleine Renouard en proposent la synthèse suivante :

«Écrire comme un enfant qui aurait trop bu » est son programme et son idéal, mais cet état d'innocence et de totale liberté a toujours été contrôlé par l'autre nécessité : celle de « trouver une phrase qui retienne tout ensemble 28 ».

L'examen de quelques aspects de cet ensemble mouvant de tentatives, d'œuvres abandonnées et de brouillons fait en effet apparaître de constantes mises en tension qui permettent de préciser cette hypothèse des deux nécessités complémentaires du relâchement et du contrôle à l'œuvre dans le travail d'écriture de Pinget. Ainsi, les pôles entre lesquels s'élabore le sens se placent-ils aussi bien entre croyance et nihilisme, revendication de la simplicité et attachement à des formes rares d'un savoir séculaire, dilection démocratique et orientation aristocratique, mélancolie et érudition imaginaire ${ }^{29}$, mais aussi entre le besoin de respirer et le désir de faire durer le récit. L'examen du laboratoire central de Robert Pinget confirme en outre que si son œuvre résiste à toute tentative de classement, c'est que, à la fois, elle est intempestive, « antimoderne », par nombre de ses références et qu'elle se situe au cœur des préoccupations de cette modernité que Malraux fait naître, avec Goya, sous le signe du sommeil de la raison qui engendre les monstres.

28. Op. cit., p. 31.

29. Voir Nathalie Piégay-Gros, L'Érudition imaginaire, Genève, Droz, 2009, p. 144-150. 
Martin Mégevand est maître de conférences à l'université Paris 8. Il s'est occupé du dépôt des manuscrits de Robert Pinget à la Bibliothèque littéraire Jacques Doucet, a publié en 2009 avec Nathalie Piégay-Gros Mahu reparle, nouvelle inédite de Pinget, aux éditions des Cendres et Robert Pinget : matériau, marges, écriture, aux Presses universitaires de Vincennes. Il travaille également sur l'œuvre de Samuel Beckett, et sur les littératures francophones, notamment théâtrales, en lien avec l'anglophonie. Il est responsable éditorial du site <www.robert-pinget.com>.

martin.megevand@univ-paris8.fr

\section{Résumés}

\section{Le laboratoire central de Robert Pinget}

«Le laboratoire central de Robert Pinget » présente quelques-uns des nombreux textes non publiés de Robert Pinget (1919-1997) conservés à la Bibliothèque littéraire Jacques Doucet. L'article s'appuie sur des extraits de chutes de Mahu ou le matériau, le premier roman publié de Robert Pinget en 1952, de « Fonfon », une nouvelle refondue dans Passacaille (1969), et d'un texte inclassable intitulé « Messire Jonas » écrit au début des années cinquante. Il s'agit de proposer des pistes méthodologiques d'étude et d'organisation de ces textes et documents, placés en relation avec l'œuvre publiée de l'auteur. L'approche choisie vise à reconstituer quelques caractéristiques fondamentales du geste esthétique de Robert Pinget, parmi lesquels l'inachèvement, l'effacement et diverses procédures de négociation. Cet article s'inscrit dans le cadre des recherches menées par Anne Herschberg Pierrot sur le style de genèse.

“The Central Laboratory of Robert Pinget" offers a presentation of some of the many unpublished texts of Robert Pinget (19191997) held at the Bibliothèque littéraire Jacques Doucet in Paris, and seeks to provide methodological elements to help organize them and study them in relation to Pinget's edited work. The article provides excerpts from abandoned short stories written for Pinget's first published novel Mahи ои le matériau (1952), of a short novel titled "Fonfon" partly recycled in Passacaglia (1969) and presents a genre-breaking text titled "Messire Jonas" written in the early 1950s. The approach aims at considering the major components of Pinget's aesthetic gesture, focusing particularly on non-completion, erasure and negotiation procedures. The article follows Anne Herschberg Pierrot's research on genesis style.

„Die zentrale Werkstatt von Robert Pinget” präsentiert einige der zahlreichen unveröffentlichten Texte Robert Pingets (1919-1997), welche in der Bibliothèque littéraire Jacques Doucet in Paris aufbewahrt sind. Der vorliegende Artikel bezieht sich auf unveröffentlichte Auszüge aus von Pinget verworfenen Texten seines ersten veröffentlichten Romans Mahи ou le matériau (1952), aus einer Novelle namens Fonfon, die sich teilweise in Passacaille (1969) wiederfindet, und aus einem anfangs der fünfziger Jahre verfassten, schwer einzuordnenden Text mit dem Titel Messire Jonas. Es sollen methodologische Aspekte dargelegt werden, die für die Untersuchung und die Organisation dieser Texte und Dokumente hilfreich sind und sie in Zusammenhang mit dem veröffentlichten Werk des Autors bringen. Ziel des gewählten Ansatzes ist es, einige grundlegende Elemente von Robert Pingets ästhetischer Geste zu rekonstruieren, darunter insbesondere die Unfertigkeit, die Löschung und verschiedene Verfahren der Verneinung. Der Artikel folgt den von Anne Herschberg Pierrot geleiteten Untersuchungen zum Stil der Genese.
“E1 laboratorio central de Robert Pinget" presenta algunos de los numerosos textos inéditos de Robert Pinget (1919-1997) guardados en la Biblioteca Literaria Jacques Doucet. Este artículo se basa en algunos fragmentos de recortes de Mahu ou le matériau, la primera novela publicada de Robert Pinget en 1952, de "Fonfon", relato refundido en Passacaille (1969), y de un texto inclasificable titulado "Messire Jonas", escrito a comienzos de los años cincuenta. Se trata de proponer pistas metodológicas de estudio y de organización de esos textos y documentos, vinculados con la obra publicada del autor. El enfoque elegido intenta reconstituir algunos componente fundamentales del gesto estético de Robert Pinget, lo inacabado, la borradura y diversos procedimientos de negociación. Este artículo se inscribe en el marco de las investigaciones llevadas a cabo por Anne Herschberg Pierrot sobre el estilo de la génesis.

O "laboratório central de Robert Pinget" apresenta alguns dos numerosos textos inéditos de Pinget (1919-1997) que estão conservados na Biblioteca Literária Jacques Doucet. O artigo baseia-se em extratos abandonados de Mahu ou le matériau, o seu primeiro romance, publicado em 1952, de "Fonfon", novela que viria a ser refundida como Passacaille (1969), e um texto inclassificável intitulado "Messire Jonas", escrito no início dos anos 50. Procurando pistas metodológicas para estudar e organizar estes textos e documentos, confrontados com a obra publicada do autor, optou-se por depreender alguns componentes fundamentais do gesto estético de Robert Pinget, tais como o inacabamento, a rasura e procedimentos de negociação. Este artigo integra-se nas pesquisas realizadas por Anne Herschberg Pierrot sobre estilo genético.

"I1 laboratorio centrale di Robert Pinget" presenta alcuni dei numerosi testi dello scrittore svizzero (1919-1997) rimasti inediti e ora conservati a Parigi nella Bibliothèque littéraire Jacques Doucet. L'articolo si fonda su estratti di Mahu ou le matériau, il primo romanzo pubblicato da Robert Pinget nel 1952, di "Fonfon", una novella confluita in Passacaille (1969), e di un testo inclassificabile intitolato "Messire Jonas", scritto nei primi anni cinquanta. L'articolo si propone, come obiettivo, di prospettare al lettore percorsi metodologici di studio e di organizzazione di questi materiali d'autore, visti in relazione ai testi e documenti invece pubblicati dall'autore. L'approccio mira a ricomporre taluni basilari componenti che caratterizzano il criterio estetico di Robert Pinget: il non-finito, la cancellazione, e i diversi sistemi di negoziazione. L'articolo si colloca entro la più ampia ricerca condotta da Anne Herschberg Pierrot intorno al tema dello stile genetico. 